\title{
Serum C1q- binding adiponectin in maintenance hemodialysis patients
}

\author{
Ken Kishida ${ }^{1,2^{*}}$, Naohiro Kishida ${ }^{2}$, Masaaki Arima ${ }^{2}$, Hideaki Nakatsuji ${ }^{1}$, Hironori Kobayashi ${ }^{3}$, Tohru Funahashi ${ }^{1,4}$ and \\ lichiro Shimomura ${ }^{1}$
}

\begin{abstract}
Background: Patients on maintenance hemodialysis (HD) have much higher levels of adiponectin (Total-APN). Adiponectin and C1q form a protein complex in human blood, and serum C1q-binding adiponectin (C1q-APN) can be measured. We recently reported that C1q-APN/Total-APN ratio rather than Total-APN correlated with atherosclerosis in diabetics. However, the characteristics of C1q-APN in HD patients remain unclear. The preset study investigated the characteristics of the adiponectin parameters including C1q-APN and also to clarify the relationship between various serum adiponectin parameters and atherosclerotic cardiovascular diseases (ACVD) in HD patients.

Methods: The single cross-sectional study subjects were 117 Japanese patients (males/females $=61 / 56$ ) on regular HD. Blood Total-APN, high molecular weight-adiponectin (HMW-APN), C1q-APN and C1q concentrations were measured by enzyme-linked immunosorbent assays. ACVD were defined as stroke, coronary and peripheral artery diseases, thoracic and abdominal aneurysms.

Results: Stepwise regression analysis identified high-density lipoprotein-cholesterol (HDL-C) as the only significant and independent determinant of C1q-APN in males, and duration of HD as the only significant and independent determinant of C1q-APN in females. Stepwise regression analysis identified uric acid, low-density lipoproteincholesterol and triglyceride as significant and independent determinants of C1q-APN/Total-APN ratio in males, and leukocyte count and HDL-C as significant and independent determinants of C1q-APN/Total-APN ratio in females. Multiple logistic regression analysis identified inorganic phosphorus and C1q-APN or C1q-APN/C1q ratio as significant determinants of ACVD.
\end{abstract}

Conclusions: Low serum C1q-APN and C1q-APN/C1q ratio, but not C1q-APN/Total-APN ratio, correlated with ACVD in HD patients.

Trial registration: ClinicalTrials.gov: UMIN 000004318

Keywords: Adiponectin, C1q, C1q-binding adiponectin, Hemodialysis

\section{Background}

Adiponectin is an adipose-specific circulating protein [1], and circulating total-adiponectin levels (Total-APN) are lower in males than in females but not different between pre- and postmenopausal females [2]. Adiponectin has protective properties against diabetes and atherosclerotic cardiovascular disease (ACVD) [3]. ACVD is

\footnotetext{
* Correspondence: kkishida@imed2.med.osaka-u.ac.jp

'Department of Metabolic Medicine, Graduate School of Medicine, Osaka University, 2-2 B-5, Yamada-oka, Suita, Osaka 565-0871, Japan

${ }^{2}$ Kishida Clinic, 5-6-3, Honmachi, Toyonaka, Osaka 560-0021, Japan

Full list of author information is available at the end of the article
}

the major cause of morbidity and mortality in maintenance hemodialysis (HD) patients. Zoccali and our group demonstrated the presence of consistently high levels of Total-APN in HD patients, and that low Total-APN levels were associated with increased risk for ACVD events in HD patients over a follow-up period of 31 months [4]. We recently reported that adiponectin binds with $\mathrm{C1q}$ in human blood, and also described the development of a system to measure human serum C1qbinding adiponectin (C1q-APN) [5]. Although circulating Total-APN level is considered a biomarker of the metabolic syndrome [6], serum C1q-APN/Total-APN

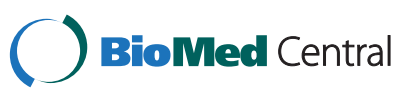


ratio rather than Total-APN is a novel marker of the metabolic syndrome in male subjects [5]. Serum C1qAPN/Total-APN correlated with polyvascular diseases and coronary artery disease in type 2 diabetics $[7,8]$. These data suggest that it is important to consider not only the absolute amount of adiponectin but also the levels of relative adiponectin forms in blood. However, the characteristics of C1q-APN, C1q-APN/Total-APN and $\mathrm{C} 1 \mathrm{q}-\mathrm{APN} / \mathrm{C} 1 \mathrm{q}$ in $\mathrm{HD}$ patients remain unclear.

The biochemical and haematological parameters should be also important factors in mortality outcomes in HD. The aim of the present study was to determine the relationship between biochemical and haematological parameters, clinical features, and various adiponectin parameters including $\mathrm{C} 1 \mathrm{q}-\mathrm{APN}$, and to clarify the relationship between various serum adiponectin parameters and ACVD, in HD patients.

\section{Methods}

\section{Participants}

The study (Victor-J study; \#UMIN 000004318) subjects were 117 Japanese patients $(\mathrm{n}$; males/females $=61 / 56)$ on standardized HD for at least 1 year $(500-800 \mathrm{~mL} / \mathrm{min}$ dialysate flow; 250-300 mL/min blood flow; 3.5-4 hours dialysis per session; 3 sessions per week), who visited the Kishida Clinic in February 2010. Patients treated with pioglitazone, which is known to increase serum adiponectin levels [9], and/or patients with clinical evidence of heart failure (defined as dyspnea in addition to two of the following conditions: increased jugular pressure, bi-basal crackles on auscultation, pulmonary venous hypertension, or interstitial edema on the chest $\mathrm{x}$ ray, requiring hospitalization or extra ultrafiltration) were excluded from the study. The Medical Ethics Committee of Osaka University approved the study. Each participant gave a written informed consent.

\section{Anthropometry and laboratory tests}

Anthropometric variables [height and weight] were measured in the standing position and body mass index (BMI) was calculated [=weight $(\mathrm{kg}) /$ height $\left.(\mathrm{m})^{2}\right]$. Waist circumference (WC) at the umbilical level was measured with a non-stretchable tape in late expiration while a standing (in $\mathrm{cm}$ ). Systolic and diastolic blood pressures (SBP, DBP) were measured with a standard mercury sphygmomanometer on the right or left arm in the supine position after at least 5-minute rest.

Venous blood samples were collected before dialysis session for measurements of serum biochemical parameters, red blood cell counts (RBC), hemoglobin $(\mathrm{Hb})$, hematocrit $(\mathrm{Ht})$, white blood cell counts (WBC), creatinine $(\mathrm{Cr})$, blood urea nitrogen $(\mathrm{BUN})$, albumin (Alb), calcium (Ca), inorganic phosphorus (IP), potassium (K), magnesium (Mg), uric acid (UA), intact parathyroid hormone (intact-PTH), $\beta 2$-microglobulin ( $\beta 2 \mathrm{MG})$, blood glucose (BS), total-cholesterol triglyceride (TG), high-density lipoprotein-cholesterol (HDL-C), and $\mathrm{C}$-reactive protein (CRP). Low-density lipoproteincholesterol (LDL-C) was calculated using the Friedewald formula. Adjusted-Ca $(\mathrm{mg} / \mathrm{dL})=$ measured serum total $\mathrm{Ca}(\mathrm{mg} / \mathrm{dL})$ - measured serum Alb (mg/dL) + 4.0. Intact-PTH was measured by immunoradiometric assay. For the purpose of the present study, serum samples that were obtained at baseline from each participant were stored promptly at $-20^{\circ} \mathrm{C}$. After thawing the samples, serum levels of Total-APN and high molecular weight-adiponectin (HMW-APN) were measured by enzyme-linked immunosorbent assay (ELISA) (Human adiponectin ELISA kit, Human HMW-adiponectin ELISA kit, Otsuka Pharmaceutical Co. Tokushima, Japan) $[1,10]$. C1q-APN and C1q were measured by our handmade ELISA, as reported previously by our group [5]. The intra- and inter-coefficients of variation for C1q-APN ELISA are below $4.6 \%$ and $6.7 \%$, respectively [5].

Hypertension (HT) was defined as SBP $\geq 140 \mathrm{mmHg}$ and/or DBP $\geq 90 \mathrm{mmHg}$, or use of antihypertensive medication; (calcium channel antagonist / angiotensin converting enzyme inhibitor or angiotensin receptor blocker $/ \beta$ blockade $/$ diuretics $/ \alpha$ blockade $=20 / 11 / 3 / 1 / 5$ ). Diabetes mellitus (DM) was defined as either fasting BS $>126 \mathrm{mg} / \mathrm{dL}$, random BS >200 mg/dL, or use of insulin (n $=11$ ) or antidiabetic medication $(\alpha G I=5)$. Dyslipidemia (DL) represented high LDL-C of $>120 \mathrm{mg} / \mathrm{dL}$, hypertriglyceridemia [fasting or postprandial TG of ${ }^{3} 150$ or $200 \mathrm{mg} / \mathrm{dL}$, respectively], and/or low HDL-C of $<40 \mathrm{mg} / \mathrm{dL}$.

\section{Measurements of baPWV, and $\mathrm{ABI}$}

Arterial stiffness was assessed by measuring brachialankle pulse wave velocity (baPWV) and ankle-brachial index (ABI) using an automatic waveform analyzer (Form/ABI; Omron-Colin Co., Komaki, Japan).

\section{Definition of ACVD}

Documented coronary artery disease (CAD) consisted of one or more of the following criteria: history of stable or unstable angina with documented CAD, history of previous myocardial infarction, percutaneous coronary intervention or coronary artery bypass graft surgery. Documented cerebrovascular disease (CVD) consisted of a hospital or neurologist report with the diagnosis of transient ischemic attack or ischemic stroke. Documented aneurysm of the thoracic aorta (TAA) consisted of aortic diameter $>4.5 \mathrm{~cm}$, and documented aneurysm of the abdominal aorta (AAA) represented aortic diameter $>4.5 \mathrm{~cm}$, an infra-renal aortic diameter $>3.0 \mathrm{~cm}$, or a history of TAA and/or AAA repair. 
Documented peripheral vascular disease (PAD) represented one or both criteria: current intermittent claudication with an ankle-brachial index of $<0.9$ or a history of intermittent claudication together with previous and related intervention, such as angioplasty, stenting, atherectomy, peripheral arterial bypass graft, or other vascular intervention, including amputation. Patients with negative results by either of the above definitions were considered ACVD-free.

\section{Statistical analysis}

Data are presented as mean \pm SEM. Data of two groups were compared by the Student's t-test. Differences in frequencies were examined by the $x^{2}$ test. Relationships

Table 1 Baseline characteristics of hemodialysis patients

\begin{tabular}{|c|c|c|c|}
\hline & Males $(n=61)$ & Females $(n=56)$ & $p$ value \\
\hline Age, years & $64 \pm 1(40-81)$ & $66 \pm 1(38-91)$ & 0.2 \\
\hline Body weight (BW), kg & $61.5 \pm 1.2(44.5-85.1)$ & $46.7 \pm 1.1(29.0-67.1)$ & $<0.0001$ \\
\hline Body mass index (BMI), kg/m2 & $22.4 \pm 0.4(17.0-31.9)$ & $20.4 \pm 0.5(12.2-27.3)$ & 0 \\
\hline Waist circumference (WC), cm & $83.6 \pm 0.9(68.8-100.0)$ & $79.0 \pm 1.4(60.0-104.0)$ & 0.01 \\
\hline Systolic blood pressure (SBP), mmHg & $146 \pm 3(83-194)$ & $137 \pm 3(105-180)$ & 0.04 \\
\hline Diastolic blood pressure (DBP), $\mathrm{mmHg}$ & $75 \pm 1(54-91)$ & $73 \pm 1(54-94)$ & 0.14 \\
\hline Duration of hemodialysis (HD), years & $11 \pm 1(1-33)$ & $11 \pm 1(1-35)$ & 0.95 \\
\hline Primary disease (CGN/Diabetic/Others) & $n=30 / 21 / 10$ & $n=31 / 10 / 15$ & 0.64 \\
\hline Hypertension $(\mathrm{HT})$ & $n=21$ & $\mathrm{n}=11$ & 0.22 \\
\hline Diabetes mellitus (DM) & $n=18$ & $n=7$ & 0.12 \\
\hline Dyslipidemia (DL) & $\mathrm{n}=8$ & $n=8$ & 0.9 \\
\hline Smoking (none-/ex-/current-smoker) & $n=12 / 34 / 15$ & $n=52 / 3 / 1$ & 0.08 \\
\hline ACVD & $n=35$ & $n=16$ & 0.03 \\
\hline (CVD/CAD/TAA+AAA/PAD) & $(n=14 / 32 / 3 / 18)$ & $(n=6 / 14 / 1 / 12)$ & \\
\hline Red blood cell count (RBC) $\times 104, / \mathrm{mL}$ & $348 \pm 7(280-532)$ & $338 \pm 4(256-406)$ & 0.19 \\
\hline Hemoglobin $(\mathrm{Hb}), \mathrm{mg} / \mathrm{dL}$ & $11.2 \pm 0.1(9.7-14.5)$ & $10.9 \pm 0.1(8.6-11.7)$ & 0.04 \\
\hline Hematocrit $(\mathrm{Ht}), \%$ & $33.6 \pm 0.4(29-45)$ & $33.0 \pm 0.2(26-36)$ & 0.25 \\
\hline White blood cell count (WBC), /mL & $5931 \pm 267(3300-10700)$ & $5711 \pm 238(3100-9600)$ & 0.54 \\
\hline Creatinine $(\mathrm{Cr}), \mathrm{mg} / \mathrm{dL}$ & $11.8 \pm 0.3(6.3-19.0)$ & $9.7 \pm 0.2(6.3-13.3)$ & $<0.0001$ \\
\hline Blood urea nitrogen (BUN), mg/dL & $65.8 \pm 2.0(32-104)$ & $67.0 \pm 2.0(37-98)$ & 0.66 \\
\hline Albumin (Alb), g/dL & $3.8 \pm 0.03(3.3-4.4)$ & $3.7 \pm 0.04(3.0-4.6)$ & 0.12 \\
\hline Adjusted-calcium (Ca), mg/dL & $9.3 \pm 0.1(8.1-11.2)$ & $9.4 \pm 0.1(8.5-10.4)$ & 0.62 \\
\hline Inorganic phosphorus (IP), mg/mL & $5.9 \pm 0.2(3.0-9.0)$ & $5.5 \pm 0.1(3.7-7.8)$ & 0.07 \\
\hline Potassium (K), mEq/L & $5.1 \pm 0.1(3.6-7.0)$ & $5.2 \pm 0.1(3.7-6.3)$ & 0.76 \\
\hline Magnesium (Mg), mg/dL & $2.9 \pm 0.1(2.1-4.1)$ & $2.9 \pm 0.1(2.1-3.8)$ & 0.74 \\
\hline Uric acid (UA), mg/dL & $7.0 \pm 0.1(5.3-9.7)$ & $7.2 \pm 0.1(5.0-9.3)$ & 0.35 \\
\hline Intact parathyroid hormone (intact-PTH), pg/mL & $114 \pm 13(9.4-412)$ & $139 \pm 14(3.2-389)$ & 0.18 \\
\hline$\beta 2$-microglobulin ( $\beta 2 M G), m g / L$ & $27.0 \pm 0.8(15.5-38.6)$ & $26.4 \pm 0.6(17.8-38.2)$ & 0.53 \\
\hline Blood glucose (BS), mg/dL & $121 \pm 5(68-302)$ & $98 \pm 4(63-239)$ & 0 \\
\hline Low-density lipoprotein-cholesterol (LDL-C), mg/dL & $82 \pm 3(32-168)$ & $83 \pm 3(35-147)$ & 0.82 \\
\hline Triglyceride (TG), mg/dL & $117 \pm 10(26-400)$ & $104 \pm 7(39-278)$ & 0.28 \\
\hline High-density lipoprotein-cholesterol (HDL-C), mg/dL & $48 \pm 2(24-92)$ & $57 \pm 2(19-87)$ & 0 \\
\hline C-reactive protein (CRP), mg/dL & $0.54 \pm 0.09(0.30-3.62)$ & $0.41 \pm 0.05(0.30-1.85)$ & 0.24 \\
\hline Ankle-brachial index (ABI) & $1.08 \pm 0.03(0.57-1.36)$ & $1.07 \pm 0.03(0.40-1.44)$ & 0.81 \\
\hline $\mathrm{baPWV}, \mathrm{cm} / \mathrm{sec}$ & $1970 \pm 77(1166-3835)$ & $2034 \pm 121(4805-5971)$ & 0.66 \\
\hline
\end{tabular}

Data are mean \pm SEM (range), or number of subjects.

$A C V D$; atherosclerotic cardiovascular diseases (stroke, coronary and peripheral artery diseases, thoracic and abdominal aneurysms), CVD; cerebrovascular disease, $C A D$; coronary artery disease, $T A A$; aneurysm of the thoracic aorta, $A A A$; aneurysm of the abdominal aorta, $P A D$; peripheral vascular disease, $C G N$; chronic glomerulonephritis. 
between two continuous variables were analyzed using scatter plots and Pearson's correlation coefficient. The correlations between clinical features and adiponectin parameters were first analyzed by simple regression analysis and then by stepwise regression analysis. The correlations between clinical features and ACVD were first analyzed by simple regression analysis and then by logistic regression analysis. In all cases, $p$ values $<0.05$ and $\mathrm{F}$ value $>4$ were considered statistically significant. All analyses were performed with the JMP Statistical Discovery Software 9.0 (SAS Institute, Cary, NC) or the Statistical Package for Social Sciences (version 11.0.1 J; SPSS, Chicago, IL).

\section{Results}

\section{Characteristics of male and female HD patients}

Table 1 summarizes the characteristics of male and female HD patients enrolled in this study. The prevalence of ACVD was significantly higher in males than females (57.4\% versus $28.6 \%, \mathrm{p}=0.0299)$. Serum levels of TotalAPN, HMW-APN and C1q-APN were significantly lower in males than in females $(16.5 \pm 1.1$ versus $23.3 \pm$ $1.3 \mu \mathrm{g} / \mathrm{mL}, \mathrm{p}=0.0001,14.5 \pm 1.2$ versus $21.5 \pm 1.5 \mu \mathrm{g} /$ $\mathrm{mL}, \mathrm{p}=0.0005,106.1 \pm 4.4$ versus $136.3 \pm 4.7$ units $/ \mathrm{mL}$, $\mathrm{p}<0.0001$, Figure 1). There was no significant difference in serum C1q level between males and females (59.7 \pm
1.2 versus $58.3 \pm 1.3 \mu \mathrm{g} / \mathrm{mL}, \mathrm{p}=0.4277$, Figure 1$)$. Serum $\mathrm{HMW}-\mathrm{APN} /$ Total-APN and $\mathrm{C} 1 \mathrm{q}-\mathrm{APN} / \mathrm{C} 1 \mathrm{q}$ ratios were significantly lower in males than in females $(0.82 \pm 0.02$ versus $0.89 \pm 0.02, \mathrm{p}=0.0272,1.81 \pm 0.08$ versus $2.37 \pm$ $0.08, \mathrm{p}<0.0001$, Figure 2). There was no significant difference in serum C1q-APN/Total-APN ratio between males and females $(8.02 \pm 0.556 .81 \pm 0.48, \mathrm{p}=0.1015$, Figure 2).

Serum HMW-APN levels correlated significantly and positively with serum Total-APN level both in males and females (Figure 3A). Serum C1q-APN levels correlated significantly and positively but weakly with serum Total-APN level both in males and females (Figure 3B). Serum C1q-APN levels also correlated significantly and positively with serum HMW-APN level both in males and females $(r=0.70, p<0.0001 ; r=0.57$, $\mathrm{p}<0.0001$, respectively, data not shown).

\section{Correlation between serum adiponectin parameters and clinical features in males}

We investigated the correlations between serum adiponectin parameters and clinical features in males (Table 2). Total-APN and HMW-APN correlated significantly and negatively with BMI, WC, WBC, Cr, UA, TG and CRP, and positively with adjusted-Ca and HDL-C. Stepwise regression analysis that included BMI, WC,

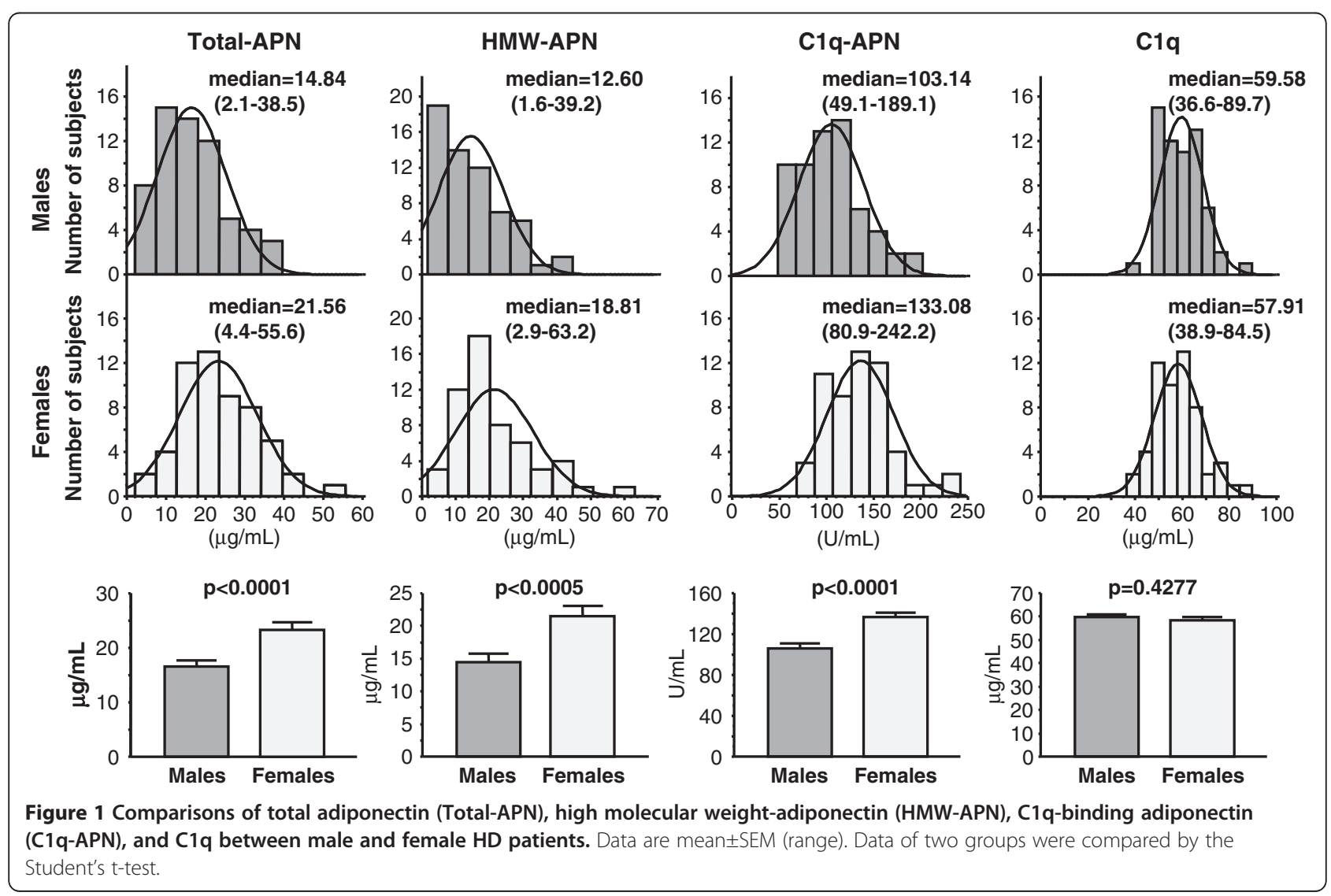


WBC, Cr, UA, adjusted-Ca, TG, HDL-C, and CRP identified UA, TG and HDL-C as significant and independent determinants of Total-APN, and TG and HDL-C as significant and independent determinants of HMWAPN. C1q-APN correlated significantly and negatively with BMI, WC, UA and TG, and positively with HDL-C. Stepwise regression analysis that included WC, WBC, UA, TG, and HDL-C identified HDL-C as the only significant and independent determinant of C1q-APN. C1q correlated significantly and negatively with age and adjusted-Ca, and positively with BMI, WC, SBP, duration of HD, WBC, IP, $\beta 2 M G$ and TG. Stepwise regression analysis that included age, WC, SBP, duration of HD, WBC, adjusted-Ca, IP, $\beta 2 M G$, and TG identified duration of HD, $\beta 2 \mathrm{MG}$ and TG as significant and independent determinants of $\mathrm{C} 1 \mathrm{q}$.

We recently showed that serum C1q-APN/Total-APN ratio is associated the metabolic syndrome [5]. We next investigated the correlations between each adiponectin ratio and clinical features in males. The HMW-APN /Total-APN ratio correlated significantly and negatively with BMI, WC, $\mathrm{Cr}$ and TG, and positively with $\mathrm{K}$ and HDL-C. Stepwise regression analysis that included WC, WBC, Cr, TG, K, and HDL-C identified HDL-C as the only significant and independent determinant of HMW-APN/Total-APN ratio. C1q-APN/Total-APN ratio correlated significantly and negatively with age, adjusted-Ca, LDL-C and HDL-C, and positively with BMI, WC, WBC, Cr, IP, UA, $\beta 2 M G, T G$ and CRP. Stepwise regression analysis that included age, WC, WBC, $\mathrm{Cr}$, adjusted-Ca, IP, UA, $\beta 2 \mathrm{MG}$, TG, HDL-C, and CRP identified Cr, UA, LDL-C and TG as significant and independent determinants of the C1q-APN/Total-APN ratio. The $\mathrm{C} 1 \mathrm{q}-\mathrm{APN} / \mathrm{C} 1 \mathrm{q}$ ratio correlated significantly and negatively with BMI, WC, WBC, UA and TG, and positively with adjusted-Ca and HDL-C. Stepwise regression analysis that included WC, WBC, adjusted-Ca, UA, TG, and HDL-C identified TG as the only significant and independent determinant of C1q-APN/C1q.

\section{Correlation between serum adiponectin parameters and clinical features in females}

Next, we investigated the correlations between serum adiponectin parameters and clinical features in females (Table 3). Total-APN correlated significantly and negatively with $\mathrm{WC}, \mathrm{WBC}$ and TG, and positively with IP and HDL-C. HMW-APN correlated significantly and negatively with WC, WBC and TG, and positively with HDL-C. Stepwise regression analysis identified WBC and HDL-C as significant and independent determinants of both Total-APN and HMW-APN. C1q-APN correlated significantly and positively with the duration of HD and IP. Stepwise regression analysis identified the duration of $\mathrm{HD}$ as the only significant and independent determinant of C1q-APN. C1q correlated significantly and negatively with UA only.

The HMW-APN/Total-APN ratio correlated significantly and negatively with WC, WBC and TG, and positively with $\mathrm{K}$. Stepwise regression analysis that included $\mathrm{WC}, \mathrm{WBC}, \mathrm{K}$, and TG identified WC and $\mathrm{K}$ as significant and independent determinants of the HMW-APN /Total-APN ratio. The $\mathrm{C} 1 \mathrm{q}-\mathrm{APN} / \mathrm{Total}-\mathrm{APN}$ ratio correlated significantly and negatively with HDL-C, and positively with WC, WBC, adjusted-Ca and TG. Stepwise regression analysis that included $\mathrm{WC}$, WBC, adjusted-Ca, TG, and HDL-C identified WBC and HDL$\mathrm{C}$ as significant and independent determinants of the $\mathrm{C} 1 \mathrm{q}-\mathrm{APN} / \mathrm{Total}-\mathrm{APN}$ ratio. The $\mathrm{C} 1 \mathrm{q}-\mathrm{APN} / \mathrm{C} 1 \mathrm{q}$ ratio correlated significantly and negatively with $\mathrm{WC}$ and TG. Stepwise regression analysis that included WC and TG identified TG as the only significant and independent determinant of the C1q-APN/C1q ratio.

\section{Simple and multivariate logistic regression analyses of ACVD}

Simple logistic regression analysis was used to evaluate the relationship between ACVD and various serum adiponectin parameters (Table 4). Sex, hypertension, smoking status (current-smoker), IP, C1q-APN, and C1q-APN/C1q correlated significantly with ACVD (Model 1; no adjustment). Multiple regression analysis identified IP and C1q-APN (Model 2) and C1q-APN /C1q ratio (Model 3) as significant determinants of ACVD in HD patients (Table 4).

\section{Discussion}

The following were the major findings of the present study in HD patients: 1) serum C1q-APN, Total-APN and HMW-APN were lower in males than in females; however, serum C1q-APN/Total-APN ratio was not different between the two sexes, 2) stepwise regression analysis identified HDL-C as the only significant and independent determinant of C1q-APN in males, and duration of $\mathrm{HD}$ as the only significant and independent determinant of C1q-APN in females, 3) stepwise regression analysis identified UA, LDL-C and TG as significant and independent determinants of the C1q-APN/TotalAPN ratio in males, and WBC and HDL-C as significant and independent determinants of the C1q-APN/TotalAPN ratio in females, and 4) multiple regression analysis identified IP and the C1q-APN or C1q-APN/C1q ratio as significant determinants of ACVD in HD patients, whereas there was no relationship between the C1qAPN/Total-APN ratio and ACVD.

Disorders of mineral metabolism, such as hyperphosphatemia, hypercalcemia, and secondary hyperparathyroidism, are independently associated with mortality and morbidity of cardiovascular diseases in HD patients 


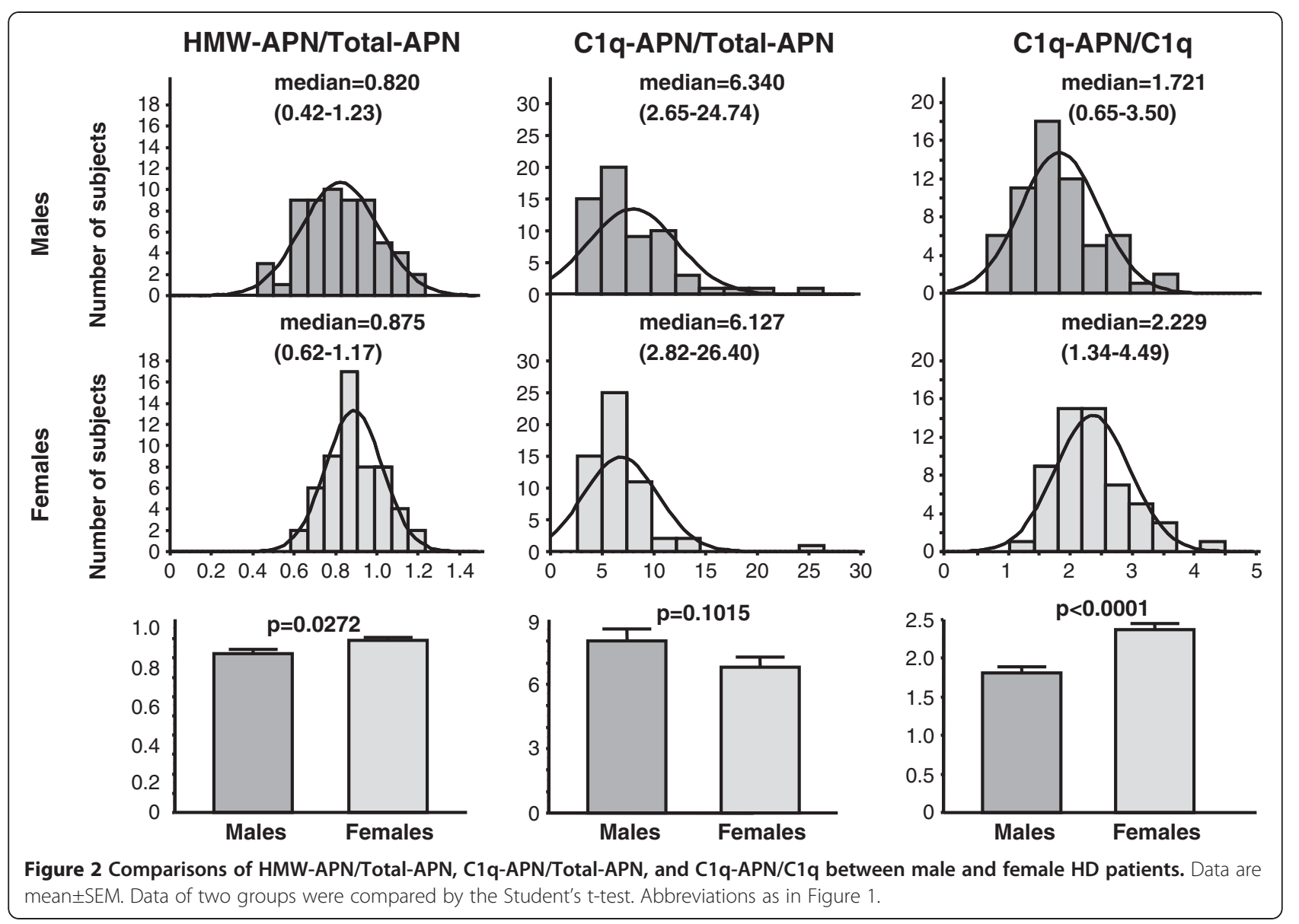

A

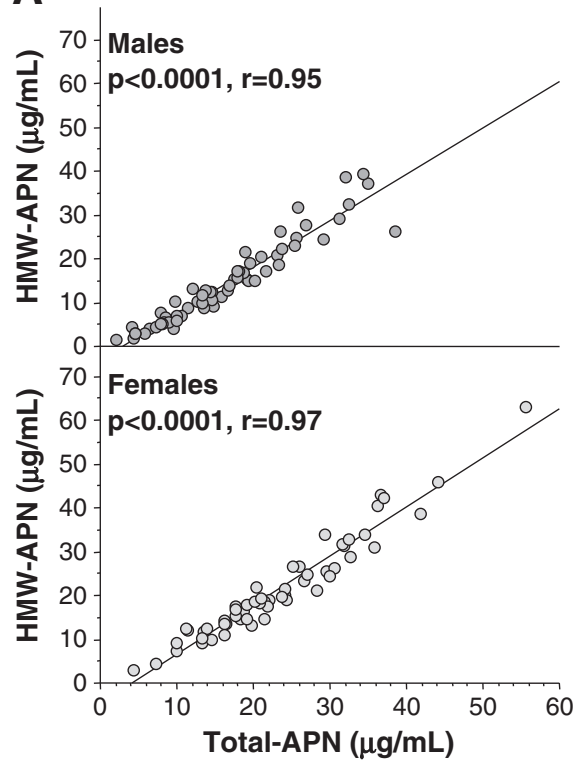

B

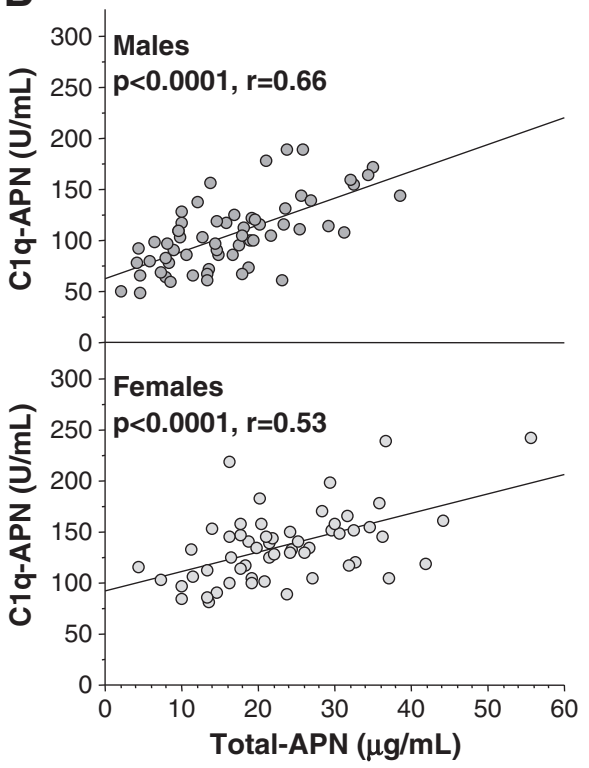

Figure 3 Correlations between HMW-APN, C1q-APN and Total-APN in male (A) and female (B) HD patients. Relationships between two continuous variables were analyzed using scatter plots and Pearson's correlation coefficient. Abbreviations as in Figure 1. 
Table 2 Correlations between various adiponectin parameters and clinical features in male patients on maintenance hemodialysis

\begin{tabular}{|c|c|c|c|c|c|c|c|c|c|c|c|c|c|c|c|c|c|c|c|c|c|}
\hline & \multicolumn{3}{|c|}{ Total-APN } & \multicolumn{3}{|c|}{ HMW-APN } & \multicolumn{3}{|c|}{ C1q-APN } & \multicolumn{3}{|c|}{$\mathrm{C1q}$} & \multicolumn{3}{|c|}{ HMW-APN/Total-APN } & \multicolumn{3}{|c|}{ C1q-APN/Total-APN } & \multicolumn{3}{|c|}{$\mathrm{C} 1 \mathrm{q}-\mathrm{APN} / \mathrm{C} 1 \mathrm{q}$} \\
\hline & \multicolumn{2}{|c|}{ Univariate } & \multirow{2}{*}{$\frac{\text { Multivariate }}{\mathrm{F}}$} & \multicolumn{2}{|c|}{ Univariate } & \multirow{2}{*}{$\frac{\text { Multivariate }}{\mathrm{F}}$} & \multicolumn{2}{|c|}{ Univariate } & \multirow{2}{*}{$\frac{\text { Multivariate }}{\mathrm{F}}$} & \multicolumn{2}{|c|}{ Univariate } & \multirow{2}{*}{$\frac{\text { Multivariate }}{\mathrm{F}}$} & \multicolumn{2}{|c|}{ Univariate } & \multirow{2}{*}{$\frac{\text { Multivariate }}{\mathrm{F}}$} & \multicolumn{2}{|c|}{ Univariate } & \multirow{2}{*}{$\frac{\text { Multivariate }}{\mathrm{F}}$} & \multicolumn{2}{|c|}{ Univariate } & \multirow{2}{*}{$\frac{\text { Multivariate }}{\mathrm{F}}$} \\
\hline & $r$ & $\mathrm{p}$ & & $r$ & $\mathrm{p}$ & & $r$ & $p$ & & $r$ & $\mathrm{p}$ & & $r$ & $p$ & & $r$ & $p$ & & $r$ & $p$ & \\
\hline Age & & 0.07 & & & 0.11 & & & 0.81 & & -0.39 & 0 & 1.7 & & 0.6 & & -0.36 & 0 & 0.15 & & 0.18 & \\
\hline BMI & -0.47 & $<0.0001$ & - & -0.4 & 0 & - & -0.27 & 0.03 & - & 0.33 & 0.01 & - & -0.28 & 0.03 & - & 0.54 & $<0.0001$ & - & -0.38 & 0 & - \\
\hline WC & -0.49 & $<0.0001$ & 0.97 & -0.42 & 0 & 0.39 & -0.28 & 0.03 & 0.49 & 0.31 & 0.02 & 2.6 & -0.28 & 0.03 & 0.75 & 0.5 & $<0.0001$ & 2.31 & -0.37 & 0 & 2.93 \\
\hline SBP & & 0.85 & & & 0.56 & & & 0.26 & & 0.27 & 0.03 & 0.02 & & 0.44 & & & 0.1 & & & 0.69 & \\
\hline $\begin{array}{l}\text { Duration of } \\
\mathrm{HD}\end{array}$ & & 0.2 & & & 0.15 & & & 0.9 & & 0.31 & 0.01 & 11.23 & & 0.3 & & & 0.09 & & & 0.43 & \\
\hline $\mathrm{Hb}$ & & 0.21 & & & 0.14 & & & 0.98 & & & 0.1 & & & 0.82 & & & 0.51 & & & 0.27 & \\
\hline $\begin{array}{l}\text { Smoking } \\
\text { (current) }\end{array}$ & & 0.29 & & & 0.43 & & & 0.28 & & & 0.62 & & & 0.1 & & & 0.15 & & & 0.43 & \\
\hline WBC & -0.43 & 0 & 0 & -0.35 & 0.01 & 0.04 & & 0.06 & & 0.37 & 0.01 & 1.1 & & 0.47 & & 0.36 & 0.01 & 3.8 & -0.39 & 0.01 & 0.7 \\
\hline $\mathrm{Cr}$ & -0.45 & 0 & 3.96 & -0.39 & 0 & 1.62 & & 0.28 & & & 0.16 & & -0.28 & 0.03 & 2.34 & 0.51 & $<0.0001$ & 7.56 & & 0.2 & \\
\hline BUN & & 0.26 & & & 0.16 & & & 0.4 & & & 0.43 & & & 0.05 & & & 0.47 & & & 0.7 & \\
\hline Alb & & 0.93 & & & 0.96 & & & 0.53 & & & 0.79 & & & 0.94 & & & 0.7 & & & 0.84 & \\
\hline adjusted-Ca & 0.33 & 0.02 & 0.84 & 0.33 & 0.02 & 0.65 & & 0.11 & & -0.3 & 0.04 & 0.89 & & 0.11 & & -0.28 & 0.05 & 0.13 & 0.32 & 0.03 & 1.51 \\
\hline IP & & 0.4 & & & 0.68 & & & 0.85 & & 0.3 & 0.04 & 0.02 & & 0.45 & & 0.32 & 0.03 & 0 & & 0.73 & \\
\hline K & & 0.13 & & & 0.07 & & & 0.93 & & & 0.68 & & 0.31 & 0.03 & 2.35 & & 0.12 & & & 0.93 & \\
\hline Mg & & 0.77 & & & 0.68 & & & 0.69 & & & 0.15 & & & 0.2 & & & 0.13 & & & 0.67 & \\
\hline UA & -0.5 & 0 & 5.13 & -0.44 & 0 & 2.46 & -0.36 & 0.01 & 3.64 & & 0.45 & & & 0.37 & & 0.4 & 0 & 4.26 & -0.34 & 0.02 & 1.65 \\
\hline Intact-PTH & & 0.52 & & & 0.26 & & & 0.29 & & & 0.06 & & & 0.14 & & & 0.09 & & & 0.69 & \\
\hline$\beta 2 M G$ & & 0.09 & & & 0.19 & & & 0.46 & & 0.43 & 0 & 14.13 & & 0.72 & & 0.37 & 0.01 & 1.15 & & 0.54 & \\
\hline BS & & 0.38 & & & 0.31 & & & 0.59 & & & 0.51 & & & 0.54 & & & 0.95 & & & 0.88 & \\
\hline LDL-C & & 0.06 & & & 0.24 & & & 1 & & & 0.66 & & & 0.92 & & -0.29 & 0.03 & 5.24 & & 0.87 & \\
\hline $\mathrm{TG}$ & -0.55 & $<0.0001$ & 10.38 & -0.5 & $<0.0001$ & 9.06 & -0.34 & 0.01 & 1.65 & 0.47 & 0 & 18.73 & -0.51 & 0.05 & 0.59 & 0.65 & $<0.0001$ & 34.07 & -0.45 & 0 & 13.46 \\
\hline HDL-C & 0.42 & 0 & 5.76 & 0.44 & 0 & 8.55 & 0.32 & 0.01 & 8.18 & & 0.08 & & 0.4 & 0 & 12.53 & -0.42 & 0 & 1.77 & 0.36 & 0 & 1.86 \\
\hline CRP & -0.31 & 0.03 & 0.04 & -0.29 & 0.04 & 0.03 & & 0.86 & & & 0.1 & & & 0.09 & & 0.33 & 0.02 & 0.49 & & 0.36 & \\
\hline
\end{tabular}

Parameters with $p<0.05$ were subsequently entered into multiple regression analysis as significant and independent variables. Parameters with $\mathrm{F}>4.0$ were significant and independent variables. Abbreviations as in Table 1. 
Table 3 Correlations between various adiponectin parameters and clinical features in female patients on maintenance hemodialysis

\begin{tabular}{|c|c|c|c|c|c|c|c|c|c|c|c|c|c|c|c|c|c|c|c|c|c|}
\hline & \multicolumn{3}{|c|}{ Total-APN } & \multicolumn{3}{|c|}{ HMW-APN } & \multicolumn{3}{|c|}{ C1q-APN } & \multicolumn{3}{|c|}{$\mathrm{C1q}$} & \multicolumn{3}{|c|}{ HMW-APN/Total-APN } & \multicolumn{3}{|c|}{ C1q-APN/Total-APN } & \multicolumn{3}{|c|}{ C1q-APN/C1q } \\
\hline & \multicolumn{2}{|c|}{ Univariate } & \multirow{2}{*}{$\frac{\text { Multivariate }}{\mathrm{F}}$} & \multicolumn{2}{|c|}{ Univariate } & \multirow{2}{*}{$\frac{\text { Multivariate }}{\mathrm{F}}$} & \multicolumn{2}{|c|}{ Univariate } & \multirow{2}{*}{$\frac{\text { Multivariate }}{\mathrm{F}}$} & \multicolumn{2}{|c|}{ Univariate } & \multirow{2}{*}{$\frac{\text { Multivariate }}{\mathrm{F}}$} & \multicolumn{2}{|c|}{ Univariate } & \multirow{2}{*}{$\frac{\text { Multivariate }}{\mathrm{F}}$} & \multicolumn{2}{|c|}{ Univariate } & \multirow{2}{*}{$\frac{\text { Multivariate }}{\mathrm{F}}$} & \multicolumn{2}{|c|}{ Univariate } & \multirow{2}{*}{$\frac{\text { Multivariate }}{\mathrm{F}}$} \\
\hline & $r$ & $\mathrm{p}$ & & $r$ & $\mathbf{p}$ & & $r$ & $\mathbf{P}$ & & $r$ & $\mathrm{p}$ & & $r$ & $p$ & & $r$ & $\mathrm{p}$ & & $r$ & $\mathbf{p}$ & \\
\hline Age & & 0.66 & & & 0.69 & & & 0.3 & & & 0.27 & & & 0.55 & & & 0.61 & & & 0.68 & \\
\hline BMI & & 0.16 & & & 0.37 & & & 0.28 & & & 0.58 & & & 0.86 & & & 0.17 & & & 0.1 & \\
\hline WC & -0.35 & 0.01 & 0.4 & -0.33 & 0.01 & 0.58 & & 0.13 & & & 0.64 & & -0.33 & 0.01 & 6.5 & 0.33 & 0.01 & 0 & -0.27 & 0.05 & 1.83 \\
\hline SBP & & 0.62 & & & 0.72 & & & 0.75 & & & 0.81 & & & 0.37 & & & 0.21 & & & 0.56 & \\
\hline Duration of $\mathrm{HD}$ & & 0.56 & & & 0.59 & & 0.31 & 0.02 & 14.43 & & 0.06 & & & 0.25 & & & 0.76 & & & 0.19 & \\
\hline $\begin{array}{l}\text { Smoking } \\
\text { (current) }\end{array}$ & & 0.46 & & & 0.33 & & & 0.32 & & & 0.27 & & & 0.23 & & & 0.86 & & & 0.69 & \\
\hline $\mathrm{Hb}$ & & 0.72 & & & 0.98 & & & 0.47 & & & 0.23 & & & 0.63 & & & 0.53 & & & 0.83 & \\
\hline WBC & -0.38 & 0.01 & 7.53 & -0.37 & 0.01 & 6.21 & & 0.13 & & & 0.66 & & -0.31 & 0.03 & 0.64 & 0.44 & 0 & 9.99 & & 0.19 & \\
\hline $\mathrm{Cr}$ & & 0.25 & & & 0.35 & & & 0.28 & & & 0.87 & & & 0.77 & & & 0.48 & & & 0.45 & \\
\hline BUN & & 0.07 & & & 0.09 & & & 0.18 & & & 0.51 & & & 0.44 & & & 0.15 & & & 0.4 & \\
\hline Alb & & 0.11 & & & 0.21 & & & 0.93 & & & 0.3 & & & 0.81 & & & 0.05 & & & 0.38 & \\
\hline adjusted-Ca & & 0.47 & & & 0.82 & & & 0.72 & & & 0.28 & & & 0.5 & & 0.32 & 0.03 & 1.35 & & 0.72 & \\
\hline IP & 0.29 & 0.05 & 0 & & 0.08 & & 0.35 & 0.02 & 2.02 & & 0.81 & & & 0.81 & & & 0.15 & & & 0.06 & \\
\hline K & & 0.41 & & & 0.19 & & & 0.16 & & & 0.59 & & 0.31 & 0.04 & 4.59 & & 0.28 & & & 0.31 & \\
\hline Mg & & 0.17 & & & 0.27 & & & 0.38 & & & 0.32 & & & 0.45 & & & 0.15 & & & 0.99 & \\
\hline UA & & 0.57 & & & 0.88 & & & 0.13 & & 0.3 & 0.04 & 4.46 & & 0.33 & & & 0.15 & & & 0.94 & \\
\hline Intact-PTH & & 0.22 & & & 0.31 & & & 0.15 & & & 0.1 & & & 0.84 & & & 0.6 & & & 0.95 & \\
\hline$\beta 2 M G$ & & 0.24 & & & 0.43 & & & 0.18 & & & 0.33 & & & 0.49 & & & 0.36 & & & 0.56 & \\
\hline BS & & 0.68 & & & 0.88 & & & 0.91 & & & 0.17 & & & 0.69 & & & 0.68 & & & 0.33 & \\
\hline LDL-C & & 0.9 & & & 0.83 & & & 0.6 & & & 0.69 & & & 0.36 & & & 0.34 & & & 0.77 & \\
\hline TG & -0.4 & 0 & 0.04 & -0.37 & 0.01 & 0.54 & & 0.23 & & & 0.07 & & -0.3 & 0.03 & 1.36 & 0.52 & $<0.0001$ & 2.37 & -0.31 & 0.02 & 5.96 \\
\hline $\mathrm{HDL}-\mathrm{C}$ & 0.55 & $<0.0001$ & 19.16 & 0.45 & 0 & 24.01 & & 0.41 & & & 0.13 & & & 0.27 & & -0.61 & $<0.0001$ & 25.35 & & 0.12 & \\
\hline CRP & & 0.92 & & & 0.87 & & & 0.73 & & & 0.16 & & & 0.79 & & & 0.73 & & & 0.38 & \\
\hline
\end{tabular}

Parameters with $\mathrm{p}<0.05$ were subsequently entered into multiple regression analysis as significant and independent variables. Parameters with $\mathrm{F}>4.0$ were significant and independent variables. Abbreviations as in Table 1. 
Table 4 Correlations between clinical features of patients on maintenance hemodialysis and ACVD

\begin{tabular}{|c|c|c|c|c|}
\hline & Model & tment & Model 2: Multivariate (C1q-APN) & Model 3: Multivariate (C1q-APN/C1q) \\
\hline & $\mathbf{r}$ & $p$ & $\mathbf{p}$ & $\mathbf{p}$ \\
\hline Age & 0.05 & 0.12 & - & - \\
\hline Sex (Male) & 0.22 & 0 & - & - \\
\hline BMl & & 0.25 & & \\
\hline WC & & 0.3 & & \\
\hline Duration of HD & & 0.53 & & \\
\hline HT & 0.12 & 0.04 & 0.53 & 0.51 \\
\hline DM & & 0.16 & & \\
\hline $\mathrm{DL}$ & & 0.28 & & \\
\hline Smoking (current) & & 0.04 & 0.53 & 0.53 \\
\hline $\mathrm{Cr}$ & & 0.63 & & \\
\hline Adjusted-Ca & & 0.73 & & \\
\hline IP & 0.2 & 0.01 & 0.01 & 0.01 \\
\hline K & & 0.61 & & \\
\hline Mg & & 0.59 & & \\
\hline UA & & 0.29 & & \\
\hline intact-PTH & & 0.53 & & \\
\hline$\beta 2 M G$ & & 0.14 & & \\
\hline CRP & & 0.19 & & \\
\hline Total-APN & & 0.13 & & \\
\hline HMW-APN & & 0.29 & & \\
\hline C1q-APN & -0.13 & 0.03 & 0.04 & \\
\hline $\mathrm{Clq}$ & & 0.43 & & \\
\hline HMW-APN/Total-APN & & 0.92 & & \\
\hline C1q-APN/Total-APN & & 0.54 & & \\
\hline Clq-APN/C1q & -0.15 & 0.02 & & 0.02 \\
\hline
\end{tabular}

Parameters with $\mathrm{p}<0.05$ in Models 2 and 3 were subsequently entered into multiple logistic regression analysis as significant and independent variables. Abbreviations as in Table 1. Model 2 (adopted factors; age, sex, HT, smoking, IP, C1q-APN). Model 3 (adopted factors; age, sex, HT, smoking, IP, C1q-APN/C1q).

[11-14]. The present study also showed that high levels of serum IP correlated with ACVD (Table 4). These results suggest that diet therapy, such as low nitrogen and phosphorus intake, are probably important for prevention of $A C V D$ in $H D$ patients. Matsubara et al. reported that increased adiponectin may contribute to the suppressive bone marrow function [15]. The present study investigated the biochemical and haematological parameters and C1q-APN paremeters. Stepwise regression analysis identified WBC counts as a significant and independent determinant of $\mathrm{C} 1 \mathrm{q}-\mathrm{APN} / \mathrm{Total}-\mathrm{APN}$ ratio in females (Table 4) but not males (Table 3). More studies are required to confirm the findings and elucidate the biological mechanisms underlying the association between the haematological parameters and C1q-APN.

Smoking and adiponectin are individually associated with cardiometabolic pathologies. A systematic review reported that there is a decreased adiponectin level in current smokers and this reduction is reversed by quitting smoking [16]. In
HD patients, smoking status (current-smoker) also correlated significantly with ACVD (no adjusted, Table 4), however there were no significant correlations between C1q-APN, C1q-APN/Total-APN, C1q-APN/C1q and smoking status in both male (Table 2) and female HD patients (Table 3). More studies are required to confirm the findings.

HD patients have much higher levels of adiponectin, compared with the general population [4]. However, low circulating levels of adiponectin independently predict cardiovascular and mortality outcomes in $\mathrm{HD}$ patients, the relationship being extensively confounded by various patient-related factors [17-22]. The cross-sectional preset study found that Total-APN did not associate with ACVD (Table 4). Complement activation and C1q binding activity have been described in HD patients [23]. Inoshita et al. [24] found significantly higher levels of functional complement activity of all three pathways, i.e., the classical pathway, the alternative pathway, and the lectin pathway, in HD patients than healthy controls. The present study showed higher 
serum C1q levels in HD male patients $(59.7 \pm 1.2 \mu \mathrm{g} / \mathrm{mL}$, Table 1) than the men of the general population $(56.0 \pm$ $10.0 \mu \mathrm{g} / \mathrm{mL}$ ) [5]. We recently reported that serum C1q-APN /Total-APN ratio correlated with the metabolic syndrome in men [5], and with polyvascular diseases and coronary artery disease in type 2 diabetics $[7,8]$, although there was no significant difference in serum C1q-APN levels between patients without and with coronary artery disease. In HD patients, low serum C1q-APN, but not C1q-APN/TotalAPN ratio, correlated with ACVD, independent of age-, sex-, other ACVD risk factors (hyperphosphatemia) (Table 4), a finding described for the first time to our knowledge. However, the different association of adiponectin-Clq with ACVD in between diabetic and HD patients remains unclear. Further studies are necessary to elucidate the pathophysiological role of C1q-APN in HD patients. Future monitoring of the long-term effects of serum C1q-APN and $\mathrm{Clq}$ on the cumulative incidence of cardiovascular events in $\mathrm{HD}$ patients is required.

The present study has several limitations. First, this is a cross-sectional study, making it difficult to establish a causeeffect relationship. Second, all patients in this study were Japanese and any differences from other ethnicities are unknown. Third, there is a potential bias in single center trials. Fourth, the number of patients was relatively small. The study included a limited number of patients and further studies of larger sample should be conducted in the future.

\section{Conclusions}

The present study demonstrated for the first time that serum C1q-APN was lower in male HD patients than in females, and that lower serum C1q-APN and C1q-APN $/ \mathrm{Clq}$ ratio, but not $\mathrm{C} 1 \mathrm{q}-\mathrm{APN} /$ Total-APN, correlated with ACVD in HD patients. These results suggest that the network of adiponectin and C1q may play a role in the pathophysiology of ACVD in HD.

\begin{abstract}
Abbreviations

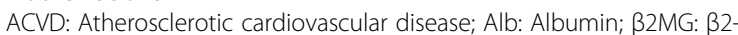
microglobulin; BMI: Body mass index; BUN: Blood urea nitrogen; BS: Blood glucose; C1q-APN: C1q-binding adiponectin; Cr: Creatinine; CRP: C-reactive protein; DBP: Diastolic blood pressure; DL: Dyslipidemia; DM: Diabetes mellitus; ELISA: Enzyme-linked immunosorbent assay; HD: Hemodialysis; HDLC: High density lipoprotein-cholesterol; HMW: High-molecular weight; HMWAPN: High molecular weight-adiponectin; intact-PTH: intact parathyroid hormone; LDL-C: Low density lipoprotein-cholesterol; RBC: Red blood cell count; SBP: Systolic blood pressure; Total-APN: Total-adiponectin; UA: Uric acid; WBC: White blood cell count; WC: Waist circumference.
\end{abstract}

\section{Competing interests}

KK, TF and IS are promotional speakers for Otsuka Pharmaceutical Co., Ltd. TF is a member of the "Department of Metabolism and Atherosclerosis", a sponsored course endowed by Kowa Co. Ltd. The company has a scientific officer who oversees the program. All other authors declare no competing interests. Human serum C1q-binding adiponectin complex assay is under patent application in Japan.

\section{Authors' contributions}

KK researched, collected, analyzed the data, participated in the concept and design of the study, interpretation of data and reviewed/edited the manuscript. NK and MA recruited the patients and collected the data. HN and HK analyzed the data.TF and IS contributed to the discussion and wrote the manuscript. All authors read and approved the final version of the manuscript.

\section{Acknowledgments}

We thank all the staff at Kishida Clinic for the excellent medical care, and Messrs Shigeo Takahashi, Suguru Akamatsu, and Tetsuya Oda for the statistical advice and helpful discussion, and Messrs Hideaki Tanaka and Tohru Hadama and Mrs. Miyuki Nakamura for the excellent technical assistance.

This research was supported in part by a Grant-in-Aid for Scientific Research on Innovative Areas (Research in a proposed research area) "Molecular Basis and Disorders of Control of Appetite and Fat Accumulation" (\#22126008, to T.F. and K.K.), and Osaka University's academia-industry collaboration policy position on collaboration between Osaka University and Otsuka Pharmaceutical Co., Ltd.

\section{Author details}

${ }^{1}$ Department of Metabolic Medicine, Graduate School of Medicine, Osaka University, 2-2 B-5, Yamada-oka, Suita, Osaka 565-0871, Japan. ${ }^{2}$ Kishida Clinic, 5-6-3, Honmachi, Toyonaka, Osaka 560-0021, Japan. 3 Department of Research and Development, Diagnostic Division, Otsuka Pharmaceutical Co., Ltd,

Tokushima 771-0195, Japan. ${ }^{4}$ Department of Metabolism and Atherosclerosis, Graduate School of Medicine, Osaka University, 2-2 B-5, Yamada-oka, Suita,

Osaka 565-0871, Japan

Received: 25 September 2012 Accepted: 22 February 2013

Published: 26 February 2013

\section{References}

1. Arita Y, Kihara S, Ouchi N, Takahashi M, Maeda K, Miyagawa J, Hotta K, Shimomura I, Nakamura T, Miyaoka K, Kuriyama H, Nishida M, Yamashita S, Okubo K, Matsubara K, Muraguchi M, Ohmoto Y, Funahashi T, Matsuzawa Y: Paradoxical decrease of an adipose-specific protein, adiponectin, in obesity. Biochem Biophys Res Commun 1996, 257:79-83.

2. Nishizawa H, Shimomura I, Kishida K, Maeda N, Kuriyama H, Nagaretani H, Matsuda M, Kondo H, Furuyama N, Kihara S, Nakamura T, Tochino Y, Funahashi T, Matsuzawa Y: Androgens decrease plasma adiponectin, an insulin-sensitizing adipocyte-derived protein. Diabetes 2002, 51:2734-2741

3. Kishida K, Funahashi T, Shimomura I: Molecular mechanisms of diabetes and atherosclerosis: role of adiponectin. Endocr Metab Immune Disord Drug Targets 2012, 12:118-131.

4. Zoccali C, Mallamaci F, Tripepi G, Benedetto FA, Cutrupi S, Parlongo S, Malatino LS, Bonanno G, Seminara G, Rapisarda F, Fatuzzo P, Buemi M, Nicocia G, Tanaka S, Ouchi N, Kihara S, Funahashi T, Matsuzawa Y: Adiponectin, metabolic risk factors, and cardiovascular events among patients with end-stage renal disease. J Am Soc Nephrol 2002, 13:134-141.

5. Nakatsuji H, Kobayashi H, Kishida K, Nakagawa T, Takahashi S, Tanaka H, Akamatsu S, Funahashi T, Shimomura I: Binding of adiponectin and C1q in human serum, and clinical significance of the measurement of C1qadiponectin / total adiponectin ratio. Metabolism 2013, 62:109-120.

6. Ryo M, Nakamura T, Kihara S, Kumada M, Shibazaki S, Takahashi M, Nagai M, Matsuzawa Y, Funahashi T: Adiponectin as a biomarker of the metabolic syndrome. Circ J 2004, 68:975-981.

7. Hirata A, Kishida K, Kobayashi H, Nakatsuji H, Funahashi T, Shimomura I: Correlation between serum C1q-adiponectin/total adiponectin ratio and polyvascular lesions detected by vascular ultrasonography in Japanese type 2 diabetics. Metabolism 2013; 62:376-385.

8. Hirata A, Kishida K, Nakatsuji H, Kobayashi H, Funahashi T, Shimomura I: High serum $\mathrm{C} 1 \mathrm{q}$-adiponectin/total adiponectin ratio correlates with coronary artery disease in Japanese type 2 diabetics. Metabolism 2012. in press.

9. Nakatsuji H, Kishida K, Kobayashi H, Funahashi T, Shimomura I: The Senri Study II Group: Three-month treatment with pioglitazone reduces circulating $\mathrm{C} 1 \mathrm{q}$-binding adiponectin complex to total-adiponectin ratio without changes in body mass index in people with type 2 diabetes. Diabetes Res Clin Pract 2013, 99:e14-e17.

10. Komura N, Kihara S, Sonoda M, Kumada M, Fujita K, Hiuge A, Okada T, Nakagawa Y, Tamba S, Kuroda Y, Hayashi N, Sumitsuji S, Kawamoto T, 
Matsumoto S, Ouchi N, Arita Y, Okamoto Y, Shimomura I, Funahashi T, Matsuzawa Y, Osaka CAD Group: Clinical significance of high-molecular weight form of adiponectin in male patients with coronary artery disease. Circ J 2008, 72:23-28.

11. Block GA, Hulbert-Shearon TE, Levin NW, Port FK: Association of serum phosphorus and calcium $\mathrm{x}$ phosphate product with mortality risk in chronic hemodialysis patients: a national study. Am J Kidney Dis 1998, 31:607-617.

12. Block GA, Klassen PS, Lazarus JM, Ofsthun N, Lowrie EG, Chertow GM: Mineral metabolism, mortality, and morbidity in maintenance hemodialysis. J Am Soc Nephrol 2004, 15:2208-2218.

13. Kimata N, Albert JM, Akiba T, Yamazaki S, Kawaguchi T, Fukuhara S, Akizawa T, Saito A, Asano Y, Kurokawa K, Pisoni RL, Port FK: Association of mineral metabolism factors with all-cause and cardiovascular mortality in hemodialysis patients: the Japan dialysis outcomes and practice patterns study. Hemodial Int 2007, 11:340-348.

14. Komaba H, Igaki N, Takashima M, Goto S, Yokota K, Komada H, Takemoto T, Kohno M, Kadoguchi H, Hirosue Y, Goto T: Calcium, phosphorus, cardiovascular events and all-cause mortality in hemodialysis patients: a single-center retrospective cohort study to reassess the validity of the Japanese Society for Dialysis Therapy guidelines. Ther Apher Dial 2008, 12:42-48.

15. Matsubara M, Namioka K, Katayose S: Relationships between plasma adiponectin and blood cells, hepatopancreatic enzymes in women. Thromb Haemost 2004, 91:360-366.

16. Kotani K, Hazama A, Hagimoto A, Saika K, Shigeta M, Katanoda K, Nakamura M: Adiponectin and smoking status: a systematic review. J Atheroscler Thromb 2012, 19:787-794.

17. Rao M, Li L, Tighiouart H, Jaber BL, Pereira BJ, Balakrishnan VS, HEMO Study Group: Plasma adiponectin levels and clinical outcomes among haemodialysis patients. Nephrol Dial Transplant 2008, 23:2619-2628

18. Okano K, Ohba T, Matsugami K, Uchida K, Nitta K, Kabaya T: Analysis of plasma adipocytokines related to clinical and laboratory data in the maintenance hemodialysis patients. Intern Med 2008, 47:1379-1386.

19. Ohashi N, Kato A, Misaki T, Sakakima M, Fujigaki Y, Yamamoto T, Hishida A: Association of serum adiponectin levels with all-cause mortality in hemodialysis patients. Intern Med 2008, 47:485-491.

20. Tamei N, Ogawa T, Ishida H, Ando Y, Nitta K: Relationship of highmolecular-weight adiponectin levels to visceral fat accumulation in hemodialysis patients. Intern Med 2010, 49:299-305.

21. Abdallah E, Waked E, Nabil M, El-Bendary O: Adiponectin and cardiovascular outcomes among hemodialysis patients. Kidney Blood Press Res 2012, 35:247-253.

22. Amira OC, Naicker S, Manga P, Sliwa K, Mia A, Raal F, Crowther NJ, Immelman RA, Olorunju S: Adiponectin and atherosclerosis risk factors in African hemodialysis patients: a population at low risk for atherosclerotic cardiovascular disease. Hemodial Int 2012, 16:59-68.

23. Dodd NJ, Vergani D, Turney JH, Parsons V, Weston MJ: Complement activation and $\mathrm{C} 1 \mathrm{q}$ binding activity in haemodialysis. Proc Eur Dial Transplant Assoc 1981, 18:300-304.

24. Inoshita H, Ohsawa I, Kusaba G, Ishii M, Onda K, Horikoshi S, Ohi H, Tomino $Y$ : Complement in patients receiving maintenance hemodialysis: functional screening and quantitative analysis. BMC Nephrol 2010, 11:34.

\section{Submit your next manuscript to BioMed Central and take full advantage of:}

- Convenient online submission

- Thorough peer review

- No space constraints or color figure charges

- Immediate publication on acceptance

- Inclusion in PubMed, CAS, Scopus and Google Scholar

- Research which is freely available for redistribution 